\title{
Intercâmbio acadêmico cultural internacional: uma experiência de crescimento pessoal e científico
}

Academic international cultural exchange: an experience of personal and scientific growth

Intercambio académico cultural internacional: una experiencia de crecimiento personal y científico

\section{Indiara Sartori Dalmolin', Eliane Ramos Pereira", Rose Mary Costa Rosa Andrade Silva"', Maria José Baltazar Gouveialv ${ }^{\mathrm{I}}$, José Júlio Sardinheiro ${ }^{\mathrm{IV}}$}

\author{
' Universidade Federal de Santa Maria, Centro de Educação Superior Norte, Curso de Enfermagem (Graduanda). \\ Santa Maria-RS, Brasil. \\ "Universidade Federal Fluminense, Escola de Enfermagem Aurora de Afonso Costa, Departamento de Enfermagem Médico- \\ Cirúrgica, Programa de Pós-Graduação Mestrado Acadêmico em Ciências do Cuidado e da Saúde. Niterói-RJ, Brasil. \\ I"' Universidade Federal Fluminense, Escola de Enfermagem Aurora de Afonso Costa, Centro de Ciências Médicas, \\ Departamento de Enfermagem, Programa de Pós-graduação Mestrado Acadêmico Ciências do Cuidado e da Saúde/UFF. \\ Niterói-RJ Brasil. \\ "v Universidade de Algarve, Escola Superior de Saúde. Faro-Algarve, Portugal.
}

\author{
Submissão: 15-12-2012 Aprovação: 19-05-2013
}

\section{RESUMO}

Este estudo objetivou descrever a experiência discente ao integrar um programa de mobilidade acadêmica internacional, durante a graduação em enfermagem. Trata-se de relato da vivência acadêmica em Portugal, na Universidade do Algarve, viabilizada pela Universidade Federal de Santa Maria, Rio Grande do Sul, Brasil. A experiência de intercâmbio realizada no período de seis meses, possibilitou a aquisição de novos conhecimentos científicos e culturais, inclusive inovações de tecnologias em saúde, desenvolvimento de pesquisa e vínculos acadêmicos. Como contribuições, espera-se estimular e intensificar a mobilidade internacional especialmente no âmbito da graduação, considerando a sua importância para o aperfeiçoamento da formação acadêmica e a excelência do ensino superior brasileiro por meio do intercâmbio científico e cultural no exterior.

Descritores: Intercâmbio Educacional Internacional; Estudantes de Enfermagem; Ensino.

\begin{abstract}
This study aimed to describe the student experience to integrate an international academic mobility program during undergraduate nursing. It is reported academic experience in Portugal, Algarve University, made possible by the Federal University of Santa Maria, Rio Grande do Sul, Brazil. The experience of exchange performed within six months, enabled the acquisition of new scientific knowledge and cultural rights, including innovations in healthcare technology, development of research and academic links. As contributions are expected to stimulate and intensify the international mobility especially at undergraduate level, considering its importance to the improvement of academic excellence and the Brazilian higher education through scientific and cultural exchange abroad.
\end{abstract}

Key words: International Educational Exchange; Students Nursing; Teaching.

\section{RESUMEN}

Este estudio objetivó describir la experiencia de los estudiantes a integrar un programa de movilidad académica internacional durante el programa de enfermería. Este es un relato de experiencia académica en Portugal, Algarve Universidad, hecho posible por la Universidad Federal de Santa Maria, Rio Grande do Sul, Brasil. El intercambio de experiencias celebrarse dentro los seis meses, permitió a la adquisición de nuevos conocimientos científicos y culturales, incluyendo las innovaciones en tecnología de salud, la investigación y el desarrollo de vínculos académicos. En las contribuciones se espera estimular e intensificar la movilidad internacional, especialmente a nivel de pregrado, teniendo en cuenta su importancia para la mejora de la excelencia académica y la educación superior brasileña a través del intercambio científico y cultural en el exterior.

Palabras clave: Intercambio Educacional Internacional; Estudiantes de Enfermería; Enseñanza.

\section{AUTOR CORRESPONDENTEＩndiara Sartori Dalmolin E-mail: indiarasartoridalmolin@yahoo.com.br}




\section{INTRODUÇÃO}

Um conceito simples aproxima a palavra intercâmbio de troca, permuta. Num sentido amplo, o intercâmbio pode ser entendido como forma de trocar informações, crenças, culturas, conhecimentos. Nesse sentido, a experiência de viver em outro país proporciona conhecer hábitos diferentes e específicos, abre novas perspectivas, auxilia na superação de dificuldades, pois o intercambista precisa se adaptar ao ambiente, enfrentar desafios e crescer ${ }^{(1)}$ sobretudo na perspectiva de fortalecimento emocional, haja vista que a distância dos laços afetivos de origem propicia a vulnerabilidade no processo de tomada de decisões da vida pessoal e profissional.

Os programas de intercâmbio buscam promover a consolidação, expansão e internacionalização da ciência e da inovação técnico-científica. É uma oportunidade de conhecer novas culturas, sistemas políticos e organizações sociais, aprender, aprimorar e/ou conhecer as variantes linguísticas de um novo idioma. Entre as inúmeras metas destes programas destaca-se a necessidade de investir na formação de pessoal altamente qualificado nas competências e habilidades necessárias para o avanço da sociedade do conhecimento; aumentar a presença de pesquisadores e estudantes de vários níveis em instituições de excelência no exterior e promover a inserção internacional das instituições brasileiras pela abertura de oportunidades semelhantes para cientistas e estudantes estrangeiros ${ }^{(2)}$

Nessa perspectiva, experiências desse tipo são extremamente relevantes já que agregam valores ao crescimento profissional e pessoal. Contudo é necessário estar preparado, pois a iniciativa requer muita paciência, abnegação e capacidade de contornar os imprevistos, a distância de familiares e amigos; as variantes culturais, de clima, de hábitos e de valores. O intercâmbio acadêmico permite proveito e vantagens que vão além do aprendizado, mas também no desenvolvimento psicológico, autoconfiança, amadurecimento, independência, capacidade de relacionar-se e, sentir-se 'um cidadão do mundo ${ }^{\prime(3)}$.

No âmbito da mobilidade internacional, cada vez mais os órgãos de fomento do Ministério da Educação (MEC) e as Instituições de Ensino Superior (IES) vêm aumentando os incentivos por meio de convênios acadêmicos e bolsas de estudos. Assim, a intensificação de atividades, no exterior, de estudantes durante a graduação e pós-graduação, principalmente após a efetivação do programa Ciência sem Fronteiras, constitui uma metodologia inovadora no ensino, e ao mesmo tempo, transpõe questões complexas como a qualidade, eficiência, prestação de contas e competição, as quais o estudante de nível superior deve agregar e estar preparado para enfrentar na futura vida profissional ${ }^{(4)}$.

Diante do exposto, o objetivo deste relato é descrever a experiência discente durante mobilidade internacional acadêmica e cultural em um programa de intercâmbio entre Brasil e Portugal. Ressaltam-se as atividades realizadas, vivências pessoais, contatos científicos e atividades desenvolvidas no período de realização deste intercâmbio, o que justifica o estudo.

\section{RELATO DA EXPERIÊNCIA}

Trata-se de um relato de experiência discente durante mobilidade acadêmica internacional em Portugal, viabilizado por meio da Universidade Federal de Santa Maria (UFSM). O intercâmbio foi realizado na modalidade de graduação, através do Programa Acordo Bilateral entre a UFSM/Brasil e a Universidade do Algarve (UAlg)/Portugal, acordo este selado no ano de 2011 entre estas duas IES. O subsídio deste programa prevê a isenção das taxas acadêmicas no espaço europeu, sem a contemplação de bolsa auxílio para despesas de moradia, transporte e alimentação(5).

O intercâmbio começou a ser pensado após contatar e conhecer verbalmente as experiências de estudantes que já haviam passado por este mundo de oportunidades, percebendo-se o quanto uma mobilidade internacional capacita os acadêmicos para a vida pessoal, social e profissional. Empreendeu-se, então, à escolha do país, analisando os programas com disponibilidade de vagas para a enfermagem, processos seletivos, documentos.

Após processo interno de seleção, a relação dos estudantes contemplados foi enviada ao Gabinete de Relações Internacionais e Mobilidade (GRIM) da UAlg, que no âmbito da internacionalização de discentes e docentes, aceita os estudantes estrangeiros desde que cumpram a matrícula nas disciplinas, com inscrição em no mínimo 30 créditos por semestre. Este órgão também se preocupa com a recepção, trâmites burocráticos e de residência, acolhimento, orientações e oferta de vagas na residência universitária, aos estudantes de outros países. Ademais, a escolha por esta universidade portuguesa condiz à qualidade dos seus cursos, professores, instalações, área geográfica e cidade ${ }^{(6)}$. Finalmente, o parecer positivo vindo da UAlg, Portugal, foi considerado uma oportunidade enriquecedora de trocas que favorecem a integração, inclusive transcultural.

Realizado num período de seis meses, de fevereiro a julho de 2012, a mobilidade configurou-se em vivências teóricas e práticas, contatos científicos e atividades desenvolvidas em Portugal as quais permitiram trocas no processo ensino-aprendizagem, além de experiências vinculadas a traços sócio-históricos e culturais que se expressam nos modos de cuidar e acolher.

A princípio, adentrar este país e universidade gerou muitas expectativas, ainda suscitou muitas dúvidas, principalmente pelo fato de não estar praticando um novo idioma. Contudo, após ter vivido essa experiência, ficou a convicção de que não poderia ter escolhido lugar melhor, pela imensa admiração, carinho e amizades recebidas e pela qualidade do ensino que me foi destinada.

\section{Aproximações do contexto sociocultural e curricular do curso de Enfermagem da Escola Superior de Saúde da Uni- versidade do Algarve}

O intercâmbio foi uma experiência singular, principalmente pelo acolhimento, admiração, qualidade de ensino, culturas, histórias e lugares magníficos para ampliar conhecimentos 
e interações transculturais da academia, mobilidade esta que, infelizmente, a mídia televisiva pouco divulga, carecendo de maior publicidade acerca das experiências extramuros da academia, oportunizadas por essa importante e inovadora estratégia do ensino superior. De modo enriquecedor, o intercâmbio permitiu ampliar a visão do diferenciado ambiente sociocultural em que estão inseridas as políticas de saúde e de educação superior do país.

Nesse aspecto, convém destacar que Portugal possui clima mediterrâneo, belas praias, espaços naturais, monumentos históricos e patrimônios internacionais. O país divide-se em cinco regiões: Norte, Centro, Lisboa e Vale do Tejo, Alentejo e Algarve. Junta-se ainda, mais duas regiões autônomas constituídas respectivamente pelos arquipélagos atlânticos de Açores e Madeira, cada uma com características físicas, estruturais, climáticas e pessoais distintas. O Algarve, onde se localiza a universidade de intercâmbio, constitui a região turística mais importante do país e uma das mais importantes da Europa; situada ao sul do país, é a terceira região mais rica de Portugal, com um PIB per capita de $86 \%$ da média europeia, sendo Faro, a capital desta região portuguesa. Além disso, entre as mais antigas cidades portuguesas contam-se Lisboa, Porto, Viseu, Braga, Coimbra, Évora, Guarda, Lamego, Silves, Faro, Lagos e Tavira com origens pré-portucalenses e detentoras de uma história urbana, fosse romana ou árabe, ou ambas.

Inserida nesse contexto sociocultural, a UAlg consiste numa instituição pública de ensino superior que conta com um corpo docente de 820 professores para mais de $10 \mathrm{mil}$ estudantes. Constitui-se de quatro campi: campus de Gambelas (Faro), campus da Penha (Faro), campus da Saúde (Faro) e campus de Portimão (Portimão). Atualmente, conta com grande infraestrutura a nível nacional e internacional, incentivando fortemente a investigação científica ${ }^{(7)}$.

O curso de Licenciatura em Enfermagem, oferecido pela Escola Superior de Saúde da Universidade do Algarve (ESSUAlg), visa à formação científica, técnica, humana e cultural do futuro enfermeiro, habilitando-o para a prestação de cuidados em enfermagem, nos diferentes níveis de prevenção, bem como para a participação na gestão de organizações de saúde, unidades ou serviços, para a participação na formação de enfermeiros e outros profissionais de saúde.

Acrescentando-se a estas funções, ressalta-se também o compromisso com o desenvolvimento e participação em projetos e programas que fortalecem o desenvolvimento da profissão. Além disso, a escola capacita enfermeiros para o trabalho no setor público e privado: em hospitais, centros de saúde, clínicas, no ensino e na investigação ${ }^{(7)}$.

O curso possui uma estrutura curricular com formação em quatro anos, com disciplinas que consistem unidades curriculares de ensino teórico e teórico-práticas além de ensino clínico. O currículo prevê, ao longo da formação, os ensinos clínicos específicos a partir do segundo período, nas áreas médica, cirúrgica, saúde materna e obstetrícia, saúde infantil e pediatria, saúde mental e psiquiatria, saúde comunitária, finalizando com o ensino clínico de integração à vida profissional no oitavo período, que inclui a elaboração de trabalho de fim de curso em articulação teoria/prática.
Em termos de disciplinas oferecidas, não há grandes divergências entre a Enfermagem no Brasil e em Portugal. Entretanto, é coerente destacar que, no país lusitano, os acadêmicos de enfermagem realizam as suas atividades no campo prático, sob supervisão direta de um enfermeiro integrante da equipe de determinada unidade, embora sob responsabilidade docente.

Isso se deve à estrutura de ensino clínico preconizada nas diretrizes de formação europeia referente ao reconhecimento das qualificações profissionais, que institui o ensino clínico como a vertente da formação em enfermagem por meio da qual o acadêmico "aprende, no seio de uma equipe e em contato direto com um indivíduo em bom estado de saúde ou doente e/ou uma coletividade, a planejar, dispensar e avaliar os cuidados de enfermagem globais requeridos, com base nos conhecimentos e competências adquiridas"(8:41); aprende não só a trabalhar em equipe, mas também a dirigir uma equipe e a organizar os cuidados de enfermagem, incluindo a educação para a saúde destinada a indivíduos e a grupos da instituição de saúde ou da coletividade ${ }^{(8)}$.

Ao analisar essa estrutura de ensino vivenciada, pode-se ressaltar alguns benefícios para a formação, ao permitir que o acadêmico desenvolva a autonomia profissional, o olhar globalizado e o senso do trabalho em equipe mais rapidamente, por serem as exigências de tal forma maiores, pelo fato de cada aluno ter um enfermeiro em exclusivo. Por outro lado, sabe-se que o maior déficit desta metodologia de ensino, diz respeito às características deficitárias nas formas subjetivas e individualizadas de avaliação de cada enfermeiro, sendo uns mais árduos e outros mais flexíveis, o que acaba por beneficiar/prejudicar os estudantes.

Contudo, o desenvolvimento da aprendizagem é também supervisionado/acompanhado pelo professor da Escola, mediante a observação do desempenho acadêmico e reuniões de trabalho com os envolvidos no processo ensino-aprendizagem: o estudante, o enfermeiro orientador da prática e o professor, a quem compete a responsabilidade da avaliação final do aluno.

Assim, no âmbito do intercâmbio, foi interessante vivenciar as articulações socioculturais nas práticas educacionais de formação do enfermeiro. Nesse sentido, corrobora-se a premissa de que, cultura, saúde e enfermagem são instâncias profundamente imbricadas, e não há como se falar de enfermagem se não considerarmos a cultura e as políticas de saúde, assim como fazer enfermagem é fazer-se dentro do contexto da saúde e da cultura ${ }^{(9)}$.

\section{Pensar, conhecer e viver um intercâmbio: transcendendo a formação tradicional}

No âmbito da mobilidade internacional destacam-se três níveis de experiência que permitem dizer que valeu a pena ter desfrutado desta atividade: a vivência teórica e prática da enfermagem no exterior; a integração em grupos de pesquisa; e a participação em congressos científicos. Em especial, a vivência teórica e prática da enfermagem portuguesa possibilitou conhecer semelhanças e diferenças desta área da saúde, entre 
o Brasil e Portugal, pois apesar da similaridade linguística, são nações de grande diferença na organização e distribuição dos programas e políticas de saúde.

Para enriquecer os conhecimentos e a prática, o curso de Enfermagem da ESSUAlg disponibilizou a realização de estágios curriculares, com duração de 35 horas semanais por um período de seis meses. Os campos práticos foram: o Hospital de Faro, nos setores de saúde mental e psiquiatria, urgência de pediatria e o serviço de obstetrícia; a Unidade de Saúde Familiar (USF) Ria Formosa, no acompanhamento e vigilância pré-natal e pós-natal; e o Centro de Saúde de Faro, onde o trabalho centralizou-se na concretização de objetivos delineados pela escola e nas perspectivas das diretrizes do Programa Nacional de Saúde Escolar da Direção Geral da Saúde de 2006.

Durante estes ensinos clínicos, foi oportuno observar o modo de cuidar dos profissionais destes locais e aprender a trabalhar em equipe, sempre focando na educação e na prevenção em saúde. Além disso, foi possível conhecer e manipular equipamentos tecnológicos de ponta, os quais no Brasil, só existem nos hospitais de grande porte e alta complexidade de algumas capitais. Sabe-se que, no enfoque teórico-prático, as experiências do intercâmbio construídas em situações reais, muito contribuem para o fortalecimento da relação com o mundo da vida(10). Desta forma, pôde-se perceber na experiência do intercâmbio, duas dimensões: uma dimensão de conhecimento e uma dimensão que é da ordem do experimentado, da implicação psicológica do sujeito.

Assim, por ocasião do aprendizado nas práticas de enfermagem do intercâmbio, foi possível integrar este tipo de estágio em Portugal e salienta-se que foi uma oportunidade de aprender, pesquisar, conviver e fazer no cuidado em enfermagem.

Também, no processo de agregação de conhecimentos através da mobilidade, é importante destacar as visitas técnicas, em grupo, realizadas na Escola Superior de Enfermagem de Coimbra e na Escola Superior de Enfermagem do Porto. Tais eventos permitiram desfrutar um mundo de histórias, acontecimento e organizações antigas. Exemplo disto cita-se a Biblioteca Joanina da Universidade de Coimbra, localizada na cidade de Coimbra, em Portugal, a qual é uma das universidades mais antigas ainda em funcionamento na Europa e no mundo e a mais antiga de Portugal(11). Assim, integrar mesmo como expectador, esta cultura, é motivador, uma experiência pós-moderna dentro de um mundo típico medieval, que possibilita enxergar de forma diferente a história. Tais perspectivas corroboram que a experiência internacional vem se apresentando como componente importante para a análise dos sistemas nacionais de educação, as estratégias familiares de diferenciação no mercado de diplomas e a formação de setores profissionais ${ }^{(12)}$.

Ainda, foi possível participar em atividades de educação em saúde junto à comunidade escolar, desenvolvendo oficinas sobre o hábito de fumar, suas consequências para a saúde, perspectivas e desafios, além de atividades de integração e estímulo ao convício social e familiar junto aos deficientes e doentes mentais no Departamento de Psiquiatria e Saúde Mental do Hospital de Faro. Desenvolveram-se estes grupos por ocasião dos ensinos clínicos, que possibilitavam facultativamente aos estudantes a organização e desenvolvimento destas atividades. Por opção, decidi tornar os espaços de funções técnicas de enfermagem, lugares de discussões sobre temáticas relevantes aos sujeitos neles inseridos e, tais grupos foram vistos de forma positiva pelos pacientes, professores e enfermeiros supervisores.

Reforça-se a premissa de que, ao utilizar-se lugares de contato com pessoas, pode-se estimular a educação em saúde integral, incluindo políticas públicas, ambientes apropriados, além dos tratamentos médicos e curativos, comprometidas com o desenvolvimento da solidariedade e da cidadania, envolvidas na melhoria da qualidade de vida e na promoção da saúde ${ }^{(13)}$.

\section{Pesquisas no exterior: fortalecimento da iniciação e ino- vação científica}

As atividades de produção de conhecimentos através de pesquisas vêm sendo desenvolvidas por grupos de pesquisadores titulados ou em formação, organizados sob a forma de Grupos de Pesquisa ${ }^{(14)}$. Os grupos de pesquisa representam hoje, a produção de conhecimentos e formação de recursos humanos em pesquisa.

No Brasil, houve um crescimento significativo dos grupos de pesquisa da área da Enfermagem com o incremento da produção, qualificação dos integrantes, bem como o fortalecimento das bases de investigação e a maior visibilidade e reconhecimento da inovação da Enfermagem ${ }^{(14)}$. Nesta dimensão, cada vez mais o mundo acadêmico é balizado pelas atividades em investigação científica e publicação dos resultados. Logo, pesquisar no exterior é de extrema importância para a construção curricular, proporciona a relação com novos métodos de coleta e análise de dados, de estruturação de projetos e contato com comitês éticos e científicos locais.

Na presente mobilidade foi possível participar ativamente de uma pesquisa, na supervisão de uma docente da ESSUAIg, que buscou identificar o grau de satisfação das usuárias nas várias fases da gravidez acompanhada em uma Unidade de Saúde Familiar (USF) da região do Algarve em Portugal. Tal investigação tratou-se de um estudo de caráter exploratório-descritivo, com abordagem quali-quantitativa. Os dados foram coletados totalizando 25 sujeitos, mediante a aplicação de um questionário com perguntas abertas e fechadas, anteriormente aplicado e adaptado pelo Centro de Estudo e Investigação em Saúde da Universidade de Coimbra para avaliar a satisfação dos usuários em relação às Unidades de Saúde Familiares. Para a análise das informações quantitativas utilizou-se estatística descritiva, o Software SPSS 15.0 e o Excel e os dados qualitativos foram analisadas à luz da Análise de Conteúdo de Bardin. Os resultados mostraram que $68 \%$ das usuárias eram de nacionalidade portuguesa, 56\% tiveram como motivo de vinda à USF, as consultas de vigilância da gravidez. Todas as mulheres mostraram-se muito ou bastante satisfeitas e os aspectos menos positivos identificados foram o tempo de espera pelas consultas, a dificuldade de contatar a USF pelo telefone e interpretar algumas informações técnicas. Ademais, os 
resultados deste estudo apontaram que, no campo da saúde, as tecnologias leves ganham dimensão no cuidado.

As etapas investigativas, de leitura, estruturação, aprovação, coleta, análise e apresentação dos resultados aos profissionais da equipe multidisciplinar, assim como, a sua apresentação e discussão, no II Encontro de Saúde Materna e Infantil desenvolvido na Escola Superior de Saúde da Universidade do Algarve, foram momentos importantes do intercâmbio, porque permitiu conhecer referenciais teóricos específicos da Europa e aprender a persistir nos objetivos estabelecidos, pois o trâmite burocrático para a participação de discentes em trabalhos de investigação na UAlg como em todo o espaço europeu, é bastante árduo. Contudo, graças à empatia da docente orientadora da pesquisa, isso foi possível durante este intercâmbio e representou um ganho significativo no desenvolvimento de habilidades em pesquisa, o que é fundamental na construção da formação acadêmica do século XXI.

Nesse sentido, torna-se fundamental destacar que, no percurso competitivo do mercado, associado às estratégias de construção de fronteiras entre as classes sociais, grupos e categorias, produz um deslocamento na definição de competências e habilidades que possam representar um ganho nas trajetórias pessoais, escolares e profissionais ${ }^{(12)}$. Em vista disso, todo tipo de atividade de desenvolvimento científico é relevante para fortalecer o conhecimento, mas também, para assegurar uma ascensão positiva no mercado de trabalho.

\section{Socialização científica e aproximação teórica: novos sa- beres nos eventos em Portugal}

Além de todas as experiências citadas, aproveitar o intercâmbio para divulgar as pesquisas produzidas na universidade de origem, torna-se uma oportunidade grandiosa para mostrar a cultura, os problemas de maior evidência e as políticas e ações desenvolvidas na perspectiva do trinômio: universidade, comunidade e serviços de saúde no Brasil.

Considera-se que a participação e apresentação de trabaIhos em congressos, simpósios ou em outros encontros científicos é importante, por ser uma oportunidade de ouvir críticas, sugestões e aperfeiçoar o trabalho, antes de publicá-lo ${ }^{(15)}$.

Ainda, a participação em eventos científicos em Portugal, permitiu conhecer grandes estudiosos e escritores nacionais e internacionais, o que favoreceu a aproximação discente especialmente ao referencial teórico das Representações Sociais.
Isso foi possível por ocasião da $11^{\text {a }}$ Conferência Internacional de Representações Sociais, realizada na cidade de Évora, oportunizando ampliar conhecimentos científicos e filosóficos e trocar palavras com os ilustres teóricos Serge Moscovici e Denise Jodelet, fundadores da Teoria das Representações Sociais.

Também, foi nesta conferência que se estabeleceram interações acadêmicas com as professoras coautoras, docentes da Universidade Federal Fluminense, Rio de Janeiro, Brasil, e a partir deste contato de intercâmbio motivaram e orientaram o presente relato para divulgação científica, com vistas à expor os inúmeros fatores positivos e o impacto cultural que este tipo de atividade propicia ao aprendizado acadêmico.

Diante de uma experiência tão construtiva, pessoal e profissionalmente, vislumbra-se a integração também em outros programas de mobilidade em nível de pós-graduação, e ampliar novas trocas de conhecimentos e fortalecer a internacionalização do ensino e da pesquisa brasileira.

\section{CONSIDERAÇÕES FINAIS}

O intercâmbio acadêmico e cultural realizado no espaço geográfico de um país ibérico caracterizou-se como uma oportunidade de aperfeiçoamento pessoal, profissional e teórico-científico durante a graduação. Para além do acréscimo de conhecimentos e vivências na enfermagem, foi um momento de construção da personalidade, de aquisição de valores sociais e culturais, de contato com pessoas diferentes dos laços afetivos comuns e de desenvolvimento de habilidades didáticas, pedagógicas e interpessoais.

Além disso, a vivência de novos horizontes, novas teorias e novas formas de se fazer enfermeiro e integrar uma equipe multiprofissional, constituem ganhos em longo prazo, agregando fatores que certamente, estimularão pequenas e constantes mudanças nas práticas diárias em saúde. Como contribuições e implicações para a enfermagem e saúde, esta produção possibilita a integração de outros programas de mobilidade, a fim de permutar conhecimentos e fortalecer a internacionalização do ensino e da pesquisa brasileira.

Espera-se, assim, contribuir no sentido de maior incentivo e agregação desta modalidade de ensino durante os cursos de graduação, com vistas a aperfeiçoar a formação acadêmica para além das disciplinas e estágios internos aos currículos pedagógicos das universidades brasileiras, consolidado saberes e inovações por meio do intercâmbio internacional.

\section{REFERÊNCIAS}

1. Souza KV. Intercâmbio educacional internacional na modalidade doutorado sanduíche em enfermagem: relato de experiência. Esc Anna Nery Rev Enferm 2008;12(2):358-63.

2. Universidade Tecnológica Federal do Paraná [homepage na internet]. Programa Ciência sem Fronteiras oferece formação no exterior a 75 mil estudantes. [acesso em 25 set 2012]. Disponível em: http://www.utfpr.edu.br/franciscobeltrao/estrutura-universitaria/diretorias/dirppg/noticias/ programa-oferece-formacao-no-exterior-a-75-mil-estudantes

3. Oliveira MG, Pagliuca LMF. Programa de mobilidade acadêmica internacional em enfermagem: relato de experiência. Rev Gaúch Enferm 2012;33(1):195-8.

4. Santiago R, Carvalho T. Mudança no conhecimento e na profissão acadêmica em Portugal. Cad Pesqui 2011;41(143):402-26.

5. Secretaria de Apoio Internacional [homepage na internet]. 
Principais oportunidades de mobilidade [acesso em 26 out 2012]. Disponível em: http://coral.ufsm.br/sai/

6. Universidade do Algarve [homepage na internet]. Bem Vindo à Universidade do Algarve [acesso em 30 out 2012]. Disponível em: http://www.ualg.pt/index. php?option $=$ com_content\&task $=$ view\&id $=25467 \&$ Ite $\operatorname{mid}=2289$ \&lang $=$ pt.

7. Universidade do Algarve. Escola Superior de Saúde. [homepage na internet]. Centro de Estudos e Desenvolvimento em Saúde (CES) [acesso em 31 out 2012]. Disponível em: http://ess.ualg.pt/home/pt/content/centros-investigacao-1

8. Jornal Oficial da União Europeia. Directiva 2005/36/CE do Parlamento Europeu e do Conselho de 7 de Setembro de 2005, relativa ao reconhecimento das qualificações profissionais. Jornal Oficial da União Europeia [periódico na internet]. 2005 [acesso em 30 out 2012]. Disponível em: http://eur-lex.europa.eu/LexUriServ/LexUriServ.do?ur $\mathrm{i}=\mathrm{OJ}: \mathrm{L}: 2005: 255: 0022: 0142: p t: P D F$

9. Silva RMCRA, Pereira ER, Santo FHE, Silva MA. Cultura, saúde e enfermagem: o saber, o direito e o fazer crítico-humano. Rev Eletrônica Enferm 2008;10(4):1165-71.

10. Jodelet D. Experiência e representações sociais. In: Menin
MSS, Shimizu AM. Experiência e representação social: questões teóricas e metodológicas. 2. ed. São Paulo: Casa do Psicólogo; 2005.

11. Expresso [homepage na internet]. Universidade de Coimbra candidata a património mundial da UNESCO [acesso em 30 out 2012]. Disponível em: http://expresso.sapo.pt/ universidade-de-coimbra-candidata-a-patrimonio-mundial-da-unesco $=\mathrm{f} 700615$

12. Mazza D. Intercâmbios acadêmicos internacionais: bolsas Capes, CNPq e FAPESP. Cad Pesqui 2009; 39(137):521-47.

13. Machado MFAS, Monteiro EMLM, Queiroz DT, Vieira NFC, Barroso MGT. Integralidade, formação de saúde, educação em saúde e as propostas do SUS - uma revisão conceitual. Ciênc Saúde Coletiva 2007; 12(2):335-42.

14. Erdmann AL, Lanzoni GMM. Características dos grupos de pesquisa da enfermagem brasileira certificados pelo CNPq de 2005 a 2007. Esc Anna Nery Rev Enferm 2008; 12(2):316-22.

15. Oliveira Filho RS, Hochman B, Nahas FX, Ferreira LM. Fomento à publicação científica e proteção do conhecimento científico. Acta Cir Bras 2005;20(Suppl.2):35-9. 